\title{
Kinetic Boltzmann approach adapted for modeling highly ionized matter created by x-ray irradiation of a solid
}

\author{
Beata Ziaja, ${ }^{1,2,{ }^{*}}$ Vikrant Saxena, ${ }^{1, \dagger}$ Sang-Kil Son, ${ }^{1}$ Nikita Medvedev, ${ }^{1}$ Benjamin Barbrel, ${ }^{3}$ \\ Bianca Woloncewicz, ${ }^{4}$ and Michal Stransky ${ }^{5}$ \\ ${ }^{1}$ Center for Free-Electron Laser Science CFEL, Deutsches Elektronen-Synchrotron DESY, Notkestrasse 85, 22607 Hamburg, Germany \\ ${ }^{2}$ Institute of Nuclear Physics, Polish Academy of Sciences, Radzikowskiego 152, 31-342 Kraków, Poland \\ ${ }^{3}$ Center for Intense Lasers and Applications (CELIA), University of Bordeaux 1, 351 Cours de la Liberation, F-33405 Talence, France \\ ${ }^{4}$ Institute of Experimental Physics, University of Gdansk, ulica Wita Stwosza 57, 80-952 Gdansk, Poland \\ ${ }^{5}$ Department of Radiation and Chemical Physics, Academy of Sciences of the Czech Republic, Na Slovance 2, 182 21 Prague, Czech Republic
}

(Received 17 December 2015; published 26 May 2016)

\begin{abstract}
We report on the kinetic Boltzmann approach adapted for simulations of highly ionized matter created from a solid by its X-ray irradiation. X rays can excite inner-shell electrons, which leads to the creation of deeply lying core holes. Their relaxation, especially in heavier elements, can take complicated paths, leading to a large number of active configurations. Their number can be so large that solving the set of respective evolution equations becomes computationally inefficient and another modeling approach should be used instead. To circumvent this complexity, the commonly used continuum models employ a superconfiguration scheme. Here, we propose an alternative approach which still uses "true" atomic configurations but limits their number by restricting the sample relaxation to the predominant relaxation paths. We test its reliability, performing respective calculations for a bulk material consisting of light atoms and comparing the results with a full calculation including all relaxation paths. Prospective application for heavy elements is discussed.
\end{abstract}

DOI: 10.1103/PhysRevE.93.053210

\section{INTRODUCTION}

Matter triggered with x-ray radiation to a highly excited state is an object of intense experimental studies with high power laser sources, in particular with free electron lasers (FELs) [1-6]. They enable one to follow the nonequilibrium path of plasma formation, also through the transient state of warm-dense matter [7-9]. The ultrashort duration of FEL pulses makes it possible to probe the unexplored regime of electronic thermalization, which takes up to several tens of femtoseconds once the exposure starts. Dedicated experiments are underway. However, the analysis of their results requires development of novel theoretical tools describing plasma formation under the strong nonequilibrium conditions. Modeling of the ionization dynamics within a bulk of material can be done efficiently with a continuum approach [10-14]. With such an approach, evolution equations in phase space are formulated for particle distributions of electrons, atoms, and ions. They are solved on a phase-space grid. As computational costs depend only on the grid size, one then avoids a direct scaling of computational costs with particle number $N^{2}$, typical for particle approaches. This feature of continuum models allows one to also treat large samples efficiently. However, for the simulation of the early, nonequilibrium stage of sample evolution, full kinetic equations should be applied. They truly follow sample evolution during this stage, in particular yielding the information on the transient electron distribution. These equations should include every active atomic configuration appearing during the sample evolution.

In this paper, we report on the kinetic approach with the atomistic Boltzmann solver [11] adapted for simulations of

\footnotetext{
*ziaja@mail.desy.de

†vikrant.saxena@desy.de
}

highly ionized matter created by the irradiation of a solid with $\mathrm{x}$-ray pulses. For the samples irradiated with VUV radiation, the number of active atomic configurations is small, as incoming photons can excite only outer-shell electrons. For the samples excited with $\mathrm{x}$ rays, this number rapidly increases, as $\mathrm{x}$ rays can excite inner-shell electrons. This leads to the creation of deeply lying core holes. Their relaxation, especially in heavier elements, can take complicated paths, leading to a large number of active configurations. Especially in heavier elements, this number can be so large that solving the set of respective equations becomes computationally inefficient. To illustrate, in carbon $(Z=6)$, the total number of possible atomic configurations within atomic bound levels $(1 s, 2 s$, and $2 p$ ) is 27 . It is then still possible to solve the set of evolution equations for all configurations. The same can be done for other light elements. However, already for the noble gas atom, neon $(Z=10)$, the total number of atomic configurations is 63 , and for the next one, $\operatorname{argon}(Z=18)$, it amounts to 1323 . This steep increase of the configuration number limits the efficiency of the kinetic approach for high- $Z$ materials, if including all active configurations.

To overcome this difficulty, the existing continuum approaches, e.g., [15], use a superconfiguration scheme which does not follow the evolution of individual configurations but instead uses a simplified set of "average" configurations [16-19]. Here, we propose an alternative approach which uses "true" atomic configurations but limits their number by restricting the sample relaxation to predominant relaxation paths. We test its reliability and computational efficiency, performing respective calculations for a bulk material consisting of carbon atoms, and comparing their results with a full calculation including all relaxation paths.

The paper is organized as follows. First, we recall the kinetic equation formalism for $\mathrm{x}$-ray irradiated finite-size samples. Second, we adjust it for simulations of an irradiated solid. With 
this scheme, we simulate irradiation of carbon bulk in the hard $\mathrm{x}$-ray regime, testing various simplified relaxation schemes including a restricted number of atomic configurations. We then compare the results with the calculation including all configurations. Finally, we present our conclusions and outlook.

\section{KINETIC EQUATIONS FOR X-RAY IRRADIATED SAMPLES}

\section{A. Finite-size sample}

In Ref. [11], dedicated Boltzmann equations for finitesize samples irradiated with soft $\mathrm{x}$ rays were formulated in the atomistic approximation. That scheme included kinetic equations for the electron distribution in phase space, as well as the kinetic equations for the atom and ground-state ion distributions up to the highest charge state present in the system, $N_{J}$, i.e.,

$$
\begin{aligned}
& \partial_{t} \rho^{(e)}(\mathbf{r}, \mathbf{v}, t)+\mathbf{v} \cdot \partial_{\mathbf{r}} \rho^{(e)}(\mathbf{r}, \mathbf{v}, t) \\
& +\frac{e}{m}[\mathbf{E}(\mathbf{r}, t)+\mathbf{v} \times \mathbf{B}(\mathbf{r}, t)] \partial_{\mathbf{v}} \rho^{(e)}(\mathbf{r}, \mathbf{v}, t) \\
& \quad=\Omega^{(e)}\left(\rho^{(e)}, \rho^{(i)}, \mathbf{r}, \mathbf{v}, t\right)
\end{aligned}
$$

for electrons, and

$$
\begin{aligned}
& \partial_{t} \rho^{(i)}(\mathbf{r}, \mathbf{v}, t)+\mathbf{v} \cdot \partial_{\mathbf{r}} \rho^{(i)}(\mathbf{r}, \mathbf{v}, t) \\
& -\frac{i e}{M}[\mathbf{E}(\mathbf{r}, t)+\mathbf{v} \times \mathbf{B}(\mathbf{r}, t)] \partial_{\mathbf{v}} \rho^{(i)}(\mathbf{r}, \mathbf{v}, t) \\
& \quad=\Omega^{(i)}\left(\rho^{(e)}, \rho^{(i)}, \mathbf{r}, \mathbf{v}, t\right)
\end{aligned}
$$

for atoms and ions $\left(i=0, \ldots, N_{J}\right)$.

The force $\mathbf{F}$ is the electromagnetic force, $\mathbf{F}(\mathbf{r}, \mathbf{v}, t)=$ $q[\mathbf{E}(\mathbf{r}, t)+\mathbf{v} \times \mathbf{B}(\mathbf{r}, t)]$, acting on electrons and ions. The electric field $\mathbf{E}$ and magnetic field $\mathbf{B}$ have two components. The first component describes the interaction of charges with external radiation. The second component describes internal electromagnetic interaction between electrons and ions. This component is a nonlocal function of electron and ion densities.

Collision terms $\Omega^{(e, i)}$ describe the change of the electron and ion densities due to short-range processes. This change may be due to (i) the creation of the secondary electrons and highly charged ions via photo- and collisional ionizations of atoms and ions, (ii) elastic and inelastic collisions of electrons and ions, (iii) the inverse bremsstrahlung process, i.e., absorptions and emissions of photons by electrons during the elastic electron-ion collisions, (iv) recombination processes, and (v) short-range electron-electron scattering modeled with the Fokker-Planck collision integral [20,21]. Details of the collision terms can be found in Refs. [11,22]. If the collision terms are neglected, Boltzmann equations (1) and (2) reduce to the Vlasov equation [20,23] describing the evolution of a collisionless plasma. The initial configuration of Eqs. (1) and (2) is given by a smooth atomic density function, $\rho^{(0)}(\mathbf{r}, \mathbf{v}, 0)$, which represents the sample at $t=0$.

As mentioned, the left-hand side comprises the electrostatic interactions: long-range Coulomb interaction between charged particles, and their interaction with the laser field. Under the conditions of ultrashort x-ray irradiation with FELs, the magnetic component of the electromagnetic interaction can be neglected, as well as the driving effect of the laser field on the electron cloud. The right-hand side includes all predominant source terms, i.e., those describing the short-range interactions of particles with each other and with the field. Depending on the irradiation wavelength, various processes can contribute to the dynamics within the irradiated sample. In the VUV and soft X-ray regime, inverse bremsstrahlung [24-26] and outer-shell photoionization dominate. In the hard $\mathrm{x}$-ray regime, inner-shell ionization has the predominant contribution to sample photoionization, with the following Auger or fluorescence decays. The inverse bremsstrahlung effect is then entirely negligible. As a result of the sample photoionization, secondary electrons are released. They ionize the sample further through collisional processes. Cascades of secondary electrons are formed. At later times, electrons thermalize and their low-energy fraction recombines. For finite-size samples, the radiation-induced ionization leads to the escape of the most energetic electrons and to the rapid sample charging. At sufficiently high doses, this triggers a spatial expansion of the sample due to the repulsive Coulomb forces between ions and to the pressure of trapped hot electrons.

The Boltzmann equations were successfully applied to describe irradiation of noble gas clusters with FEL radiation in VUV and soft x-ray regime [27,28], and the evolution of warmdense hydrogen [29]. In order to facilitate calculations-as atomic clusters can be, with a good accuracy, approximated as spherically symmetric samples-the applied Boltzmann equation was rewritten in spherical coordinates. Angular moment expansion in the leading order was used for electron distribution. The equations were then solved for the isotropic and first transport component of the electron distribution. For ions, their radial movement was assumed, and the equations were accordingly modified. In this way, the evolution of irradiated clusters could be efficiently followed through all of its stages: nonequilibrium evolution of electron and ion distribution up to the beginning of expansion.

\section{B. Solid irradiated with high-fluence $x$-ray pulse}

High-fluence $\mathrm{x}$-ray pulses enable one to achieve transiently homogeneous ionization conditions within a large volume inside an irradiated solid. Comparing with the simulations of finite samples for which particle distributions in full phase space should be followed, such x-ray triggered, spatially uniform ionization dynamics within the bulk material, far from the material surface, allows for a significant simplification of the kinetic equations. One can then assume that both electron and ion distributions are approximately uniform within the bulk, i.e., the evolution of those distribution is position independent:

$$
\partial_{\mathbf{r}} \rho^{(e)}(\mathbf{r}, \mathbf{v}, t)=0, \quad \partial_{\mathbf{r}} \rho^{(i)}(\mathbf{r}, \mathbf{v}, t)=0 .
$$

Consequently, the quasineutrality condition is naturally imposed on the sample, as at each point the number of created electrons and ions is identical. The electrostatic force responsible for mutual interactions of electrons and ions is then always equal to 0 . The equations still account for the fast electron thermalization, as the short-range electron-electron interactions described by the Fokker-Planck term in velocity 
space remain unaffected. Second, any directionality imposed by the initial ionization processes is quickly lost, due to the large number of isotropic collisional ionization, occurring soon after the exposure starts. In this way, one can with a good accuracy restrict to the isotropic component of electron and ion distributions (in velocity). These simplifications extensively speed up calculations, as no adaptive stability condition for spatial expansion then restricts computational time steps. The simplified equations can then be conveniently applied to analyze ionization dynamics within a bulk material after its $\mathrm{x}$-ray irradiation.

\section{Hard x-ray regime}

The difficulty in applying the kinetic equations in a hard $\mathrm{x}$-ray regime lies in the large number of active atomic configurations that the system may enter, while relaxing from a deeply lying core hole state, created by a hard x-ray photon. In contrast to the soft $\mathrm{x}$-ray case discussed in [11], the kinetic equations in a hard $\mathrm{x}$-ray regime must now account not only for ground-state configurations but for all active configurations (with core holes). The number of active atomic configurations and the complexity of relaxation paths strongly increases with the increasing atomic number. Their description is already complex enough on the level of direct photoinduced processes only. In this case, one can select individual pathways through the electronic configuration space with a Monte Carlo approach [30].

However, collisional processes triggered by secondary electrons mix up between various configurations. Even if we restrict only to the predominant collisional ionization which excites a secondary electron from the outermost shell, this collisional process "switches" to various additional configurations, making the relaxation path even more complicated. Also, collisional rates depend on the transient electron and ion distributions. Therefore, such Monte Carlo scheme as proposed in [30] for photoinduced processes cannot be used in this case. A full calculation requires one to solve a set of equations for each atomic configuration. The large number of such equations, strongly increasing with the increasing $Z$, soon makes such simulations numerically intractable.

However, various relaxation paths have different probabilities. Below we develop a systematic approach to restrict the number of relaxation paths to the most probable ones. We test it in the case of irradiated carbon $(Z=6$, with 27 atomic configurations) for which the full calculation including all configurations is still computationally feasible, and one can compare the results of the full calculation with the simplified schemes.

\section{RESULTS}

Using the XАTOM package [31-33] for ab initio calculations of photoionization cross sections and decay rates, we first calculated a set of all possible transitions triggered by $\mathrm{x}$ ray photons, including photoionizations and Auger decays. Fluorescent decays can be neglected for light elements due to their very low rate $[34,35]$. We considered two energies of incoming photons: 1000 and $5000 \mathrm{eV}$. There are 74 atomic transitions for carbon atom induced by photons of those

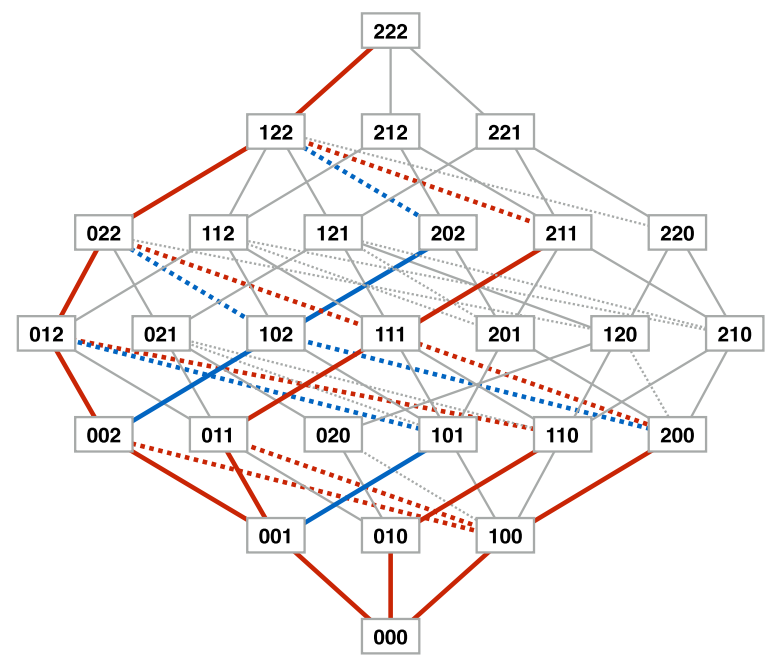

FIG. 1. All electronic configurations (27 boxes) and all possible direct photoinduced transitions (74 lines) for x-ray irradiated atomic carbon. Solid lines correspond to photoionization processes and dashed lines to Auger decays. The 19 transitions included in scheme (i) are depicted in red. Blue lines represents the additional 7 transitions added to scheme (ii), which yields 26 transitions in total.

energies. They are used for the full calculation of ionization dynamics within x-ray irradiated carbon bulk. For simplified calculations, we define two schemes:

Scheme (i). This scheme includes the most probable photoionization and the most probable Auger decay from each configuration within the path. It yields (initially) 19 photoinduced transitions, and allows for a few branches of transition chains.

Scheme (ii). In addition to scheme (i), this scheme also includes the next probable photoionization and Auger decay if they are of the same order as the most probable ones. The second scheme yields 26 photoinduced transitions for carbon.

The chain of transitions obtained is presented in Fig. 1. Carbon configurations $1 s^{n_{1}} 2 s^{n_{2}} 2 p^{n_{3}}$ are denoted as $n_{1}, n_{2}, n_{3}$, with $n_{1}, n_{2}, n_{3}=0,1,2$.

Figure 2 shows the time evolution of the average charge in these three cases for carbon bulk irradiated with a Gaussian $\mathrm{X}$-ray FEL pulse of FWHM $=10 \mathrm{fs}$. The temporal pulse maximum is at $20 \mathrm{fs}$. Maximal intensity lies within the regime typically used for high-energy-density experiments: $I_{\max }=10^{16} \mathrm{~W} / \mathrm{cm}^{2}$ for photon energy of $1000 \mathrm{eV}$, and $I_{\max }=$ $10^{18} \mathrm{~W} / \mathrm{cm}^{2}$ for photon energy of $5000 \mathrm{eV}$. The pulse fluence is then so high that approximately one atom in 20 atoms and in 100 atoms is photoionized in the two cases, respectively. Actually, only photoionization and Auger processes are considered. One can see that the relative discrepancy between the full calculation and the simplified schemes for the average charge is the largest at the end of the pulse (i.e., at $\sim 40 \mathrm{fs}$ ), and it decreases at later times. The final discrepancy is of the order of $10 \%$ for a $1000 \mathrm{eV}$ photon pulse, and of the order of $6 \%$ for a $5000 \mathrm{eV}$ photon pulse. The average energy absorbed per atom was $\sim 29 \mathrm{eV}$ for a $1000 \mathrm{eV}$ photon pulse, and $\sim 25 \mathrm{eV}$ for a $5000 \mathrm{eV}$ photon pulse (full calculation). Simplified schemes decreased the total energy absorption (per atom) in both cases by $6-7 \%$ at most. 
(a)

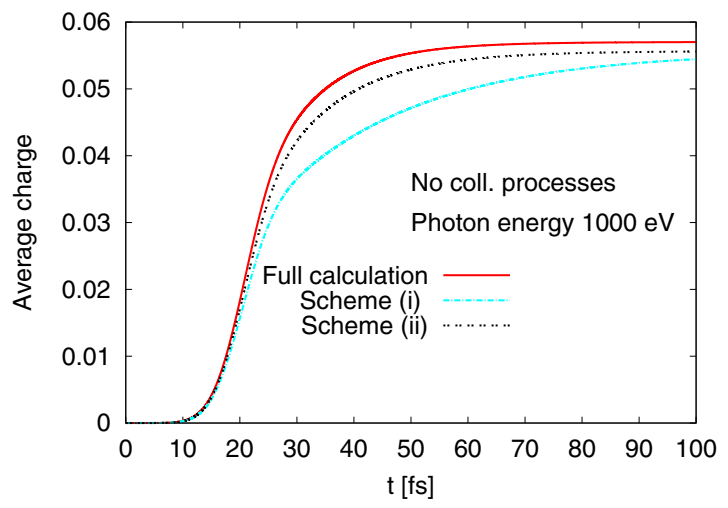

(c)

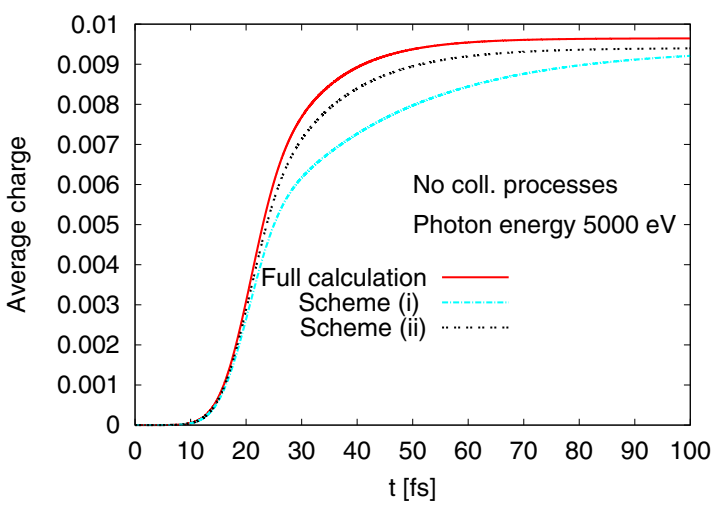

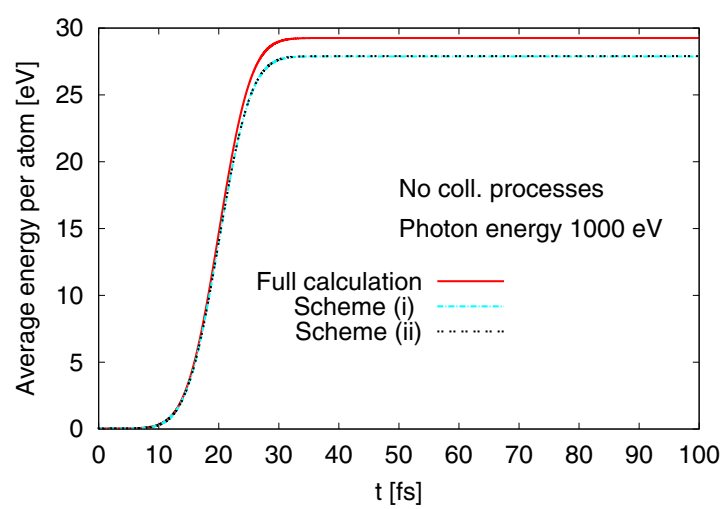

(b)

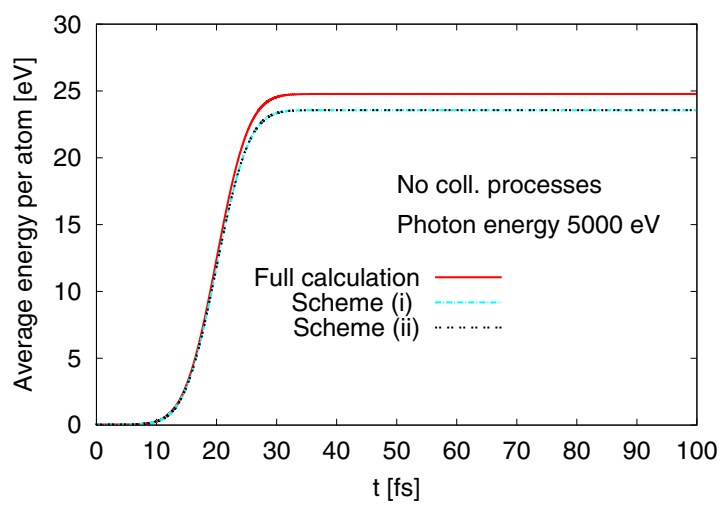

FIG. 2. Average charge and average energy absorbed per atom within carbon bulk irradiated with a Gaussian pulse of (a),(b) soft x rays $\left(E_{\text {photon }}=1000 \mathrm{eV}, I_{\max }=10^{16} \mathrm{~W} / \mathrm{cm}^{2}\right)$ and (c),(d) hard x rays $\left(E_{\text {photon }}=5000 \mathrm{eV}, I_{\max }=10^{18} \mathrm{~W} / \mathrm{cm}^{2}\right)$. Pulse maximum was at 20 fs. Only photoionization and Auger processes were considered.

These proposed schemes have then been extended to include collisional processes. Consistently with the treatment of only the predominant photoinduced processes, we include into the code only the predominant collisional ionization processes (from the outermost shell of all considered atoms and ions) and the corresponding three-body recombination rates. Let us emphasize that including collisional processes extends the number of active configurations by those that can be obtained from each "photoinduced" atomic configuration by a sequence of collisional ionization or recombination from outermost atomic shells. This forms a ladder of additional active configurations with charges from 0 to the highest one allowed in the system for each active configuration obtained through a photo- or Auger ionization. Total cross sections for electron impact ionization are taken from experiment [36]. Similarly as in Refs. [15,27,37], three-body recombination was modeled as an inverse process of collisional ionization. It is then also applicable under the conditions of nonlocal thermodynamic equilibrium (NLTE). Consistent with the description of collisional ionization, we assumed that threebody recombination brings the electron back to the outermost shell available.

As the collisional processes extend the set of active configurations besides that one defined by possible direct photoinduced transitions, we accordingly extended our scheme by including the predominant photoinduced transitions between the additional configurations accessible through collisional processes. In this way, we constructed a closed system of active configurations which can relax between each other both through photoinduced and collisional processes. The results obtained are presented below.

Figure 3 shows the time evolution of the average charge in the three calculation schemes for carbon bulk irradiated with the x-ray FEL pulse of the same parameters as in Fig. 2. Now collisional processes are included. One can see that the relative discrepancy between the full calculation and simplified schemes further decreases, remaining on the order of $7-8 \%$ at all times. However, as the full calculation includes all Auger relaxation channels, it alone shows a characteristic increase of the average charge at $\sim 90 \mathrm{fs}$. This is due to the created long-living core-hole configurations with charge +1 and +2 at the end of the pulse, their late Auger decay at 70-85 fs, and the subsequent cascading of the emitted Auger electrons of initial energy $\sim 300 \mathrm{eV}$ [Fig. 3(a)]. As expected, the total energy absorption did not change significantly in comparison with the case when collisional processes were not allowed, as the predominant photoabsorption channels had already been accounted for.

\section{DISCUSSION}

The results obtained indicate that the concept of reducing the number of active configurations by restricting to the predominant photoinduced and collisional transitions and to the participating configurations can be an efficient alternative for the calculation of material relaxation after x-ray irradiation. For carbon, the reduction of configuration number leads only to a $<10 \%$ decrease of average charge and total energy absorbed when compared to the results of full calculation, at much 

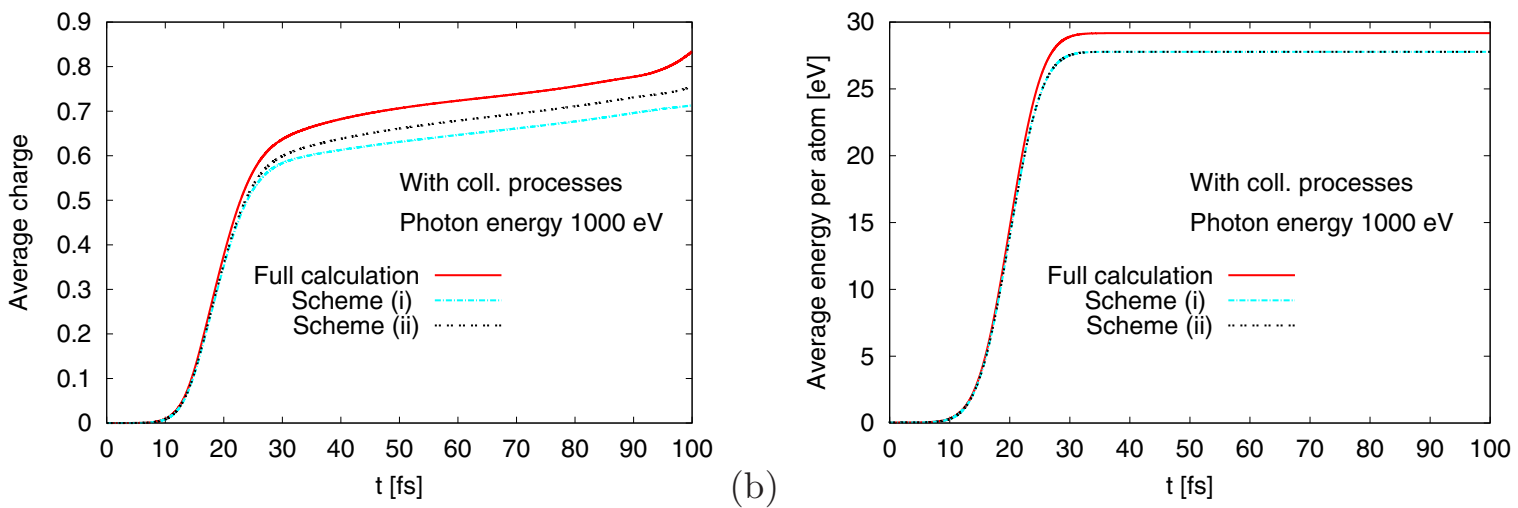

(a)

(b)

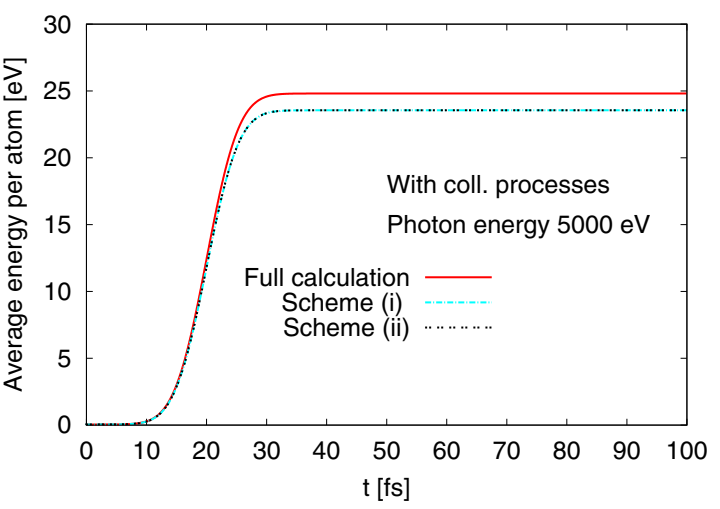

(c)

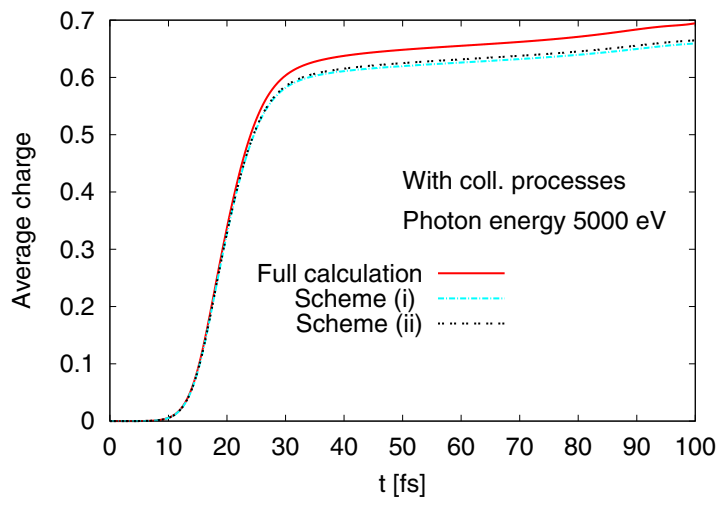

(d)

FIG. 3. Average charge and average energy absorbed per atom within carbon bulk irradiated with a Gaussian pulse of (a),(b) soft x rays $\left(E_{\text {photon }}=1000 \mathrm{eV}, I_{\max }=10^{16} \mathrm{~W} / \mathrm{cm}^{2}\right)$ and $(\mathrm{c}),(\mathrm{d})$ hard $\mathrm{x}$ rays $\left(E_{\text {photon }}=5000 \mathrm{eV}, I_{\max }=10^{18} \mathrm{~W} / \mathrm{cm}^{2}\right)$. Pulse maximum was at $20 \mathrm{fs}$. Collisional processes were included.

reduced computational costs (up to five times). Evolution time scales remain almost unaffected.

Here, only the case of a light element (carbon) was analyzed; however, the generalization of this approach to heavier elements is possible. It could then be applied for interpretation of data from warm-dense-matter (WDM) experiments with $\mathrm{x}$-ray FELs. They frequently involve heavy metals, which, due to the high number of bound electrons, can efficiently evolve to a highly excited state. For relaxation processes, fluorescent decays should be taken into account as well as Auger decays. Generalization of the proposed scheme for heavy elements would then require an efficient strategy regarding how to "sort out" the large number of possible relaxation processes (strongly increasing with $Z$ ) and the corresponding accuracy studies. The degeneracy effects in solids can be incorporated by employing the Fermi-Dirac distribution in the atomic electronic-structure calculation [33]. To sum up, such efficient calculation scheme for the relaxation processes following $x$-ray irradiation of high- $Z$ materials can enable an insight into the complex nonequilibrium dynamics triggered by $\mathrm{x}$-ray pulses, with a significant impact on the entire area of warm-dense-matter research. Corresponding investigations for a few high- $Z$ WDM experiments, using the proposed approach, are already in progress.

The code can also be applied in the VUV and optical regime $[11,27]$. Modeling of simultaneous irradiation by a two-color light source is not possible with the current version of the code. Therefore, it can be used to simulate pump-probe experiments with nonoverlapping pump and probe pulses. However, an extension of the code enabling one to treat simultaneous twocolor irradiation is possible. The extended code could then be applied for the experiments using a pump-probe scheme and high harmonics in a broad wavelength regime.

\section{ACKNOWLEDGMENTS}

The authors thank Hyun-Kyung Chung, Siegfried Glenzer, Zoltan Jurek, and Robin Santra for valuable discussions. M.S. appreciates financial support from the Czech Ministry of Education (Grant No. LG13029).
[1] P. Emma et al., First lasing and operation of an angstromwavelength free-electron laser, Nat. Photon. 4, 641 (2010).

[2] T. Ishikawa et al., A compact x-ray free-electron laser emitting in the sub-angstrom region, Nat. Photon. 6, 540 (2012).

[3] B. Rudek et al., Ultra-efficient ionization of heavy atoms by intense x-ray free-electron laser pulses, Nat. Photon. 6, 858 (2012).
[4] B. F. Murphy et al., Femtosecond X-ray-induced explosion of $\mathrm{C}_{60}$ at extreme intensity, Nat. Commun. 5, 4281 (2014).

[5] T. Gorkhover et al., Nanoplasma Dynamics of Single Large Xenon Clusters Irradiated with Superintense X-ray Pulses from the Linac Coherent Light Source Free-Electron Laser, Phys. Rev. Lett. 108, 245005 (2012). 
[6] T. Tachibana et al., Nanoplasma formation by high intensity hard x-rays, Sci. Rep. 5, 10977 (2015).

[7] S. M. Vinko et al., Creation and diagnosis of a solid-density plasma with an X-ray free-electron laser, Nature (London) $\mathbf{4 8 2}$, 59 (2012).

[8] U. Zastrau et al., Equilibration dynamics and conductivity of warm dense hydrogen, Phys. Rev. E 90, 013104 (2014).

[9] A. Lévy et al., The creation of large-volume, gradient-free warm dense matter with an x-ray free-electron laser, Phys. Plasmas 22, 030703 (2015).

[10] S. Ethier and J. P. Matte, Electron kinetic simulations of solid density Al plasmas produced by intense subpicosecond laser pulses. I. Ionization dynamics in 30 femtosecond pulses, Phys. Plasmas 8, 1650 (2001).

[11] B. Ziaja, A. R. B. de Castro, E. Weckert, and T. Möller, Modelling dynamics of samples exposed to free-electron-laser radiation with Boltzmann equations, Eur. Phys. J. D 40, 465 (2006).

[12] M. Sherlock, E. G. Hill, and S. J. Rose, Kinetic simulations of the heating of solid density plasma by femtosecond laser pulses, High Energy Density Phys. 9, 38 (2013).

[13] S. P. Hau-Riege, R. A. London, and A. Szöke, Dynamics of biological molecules irradiated by short X-ray pulses, Phys. Rev. E 69, 051906 (2004).

[14] O. Peyrusse, Coupling of detailed configuration kinetics and hydrodynamics in materials submitted to x-ray free-electronlaser irradiation, Phys. Rev. E 86, 036403 (2012).

[15] H.-K. Chung, M. H. Chen, W. L. Morgan, Y. Ralchenko, and R. W. Lee, FLYCHK: Generalized population kinetics and spectral model for spectroscopic analysis for all elements, High Energy Density Phys. 1, 3 (2005).

[16] Y. T. Lee, A model for ionization balance and L-shell spectroscopy of non-LTE plasmas, J. Quantum Spectrosc. Radiat. Transfer 38, 131 (1987).

[17] R. Marchand, S. Caille, and Y. T. Lee, Improved screening coefficients for the hydrogenic ion model, J. Quantum Spectrosc. Radiat. Transfer 43, 149 (1990).

[18] S. B. Hansen, J. Bauche, and C. Bauche-Arnoult, Superconfiguration widths and their effects on atomic models, High Energy Density Phys. 7, 27 (2011).

[19] J. Abdallah, J. Colgan, and N. Rohringer, Time-dependent calculations of electron energy distribution functions for neon gas in the presence of intense XFEL radiation, J. Phys. B 46, 235004 (2013).

[20] I. P. Sharkofsky, T. W. Johnston, and M. P. Bachynski, The Particle Kinetics of Plasmas (Addison Wesley, Reading, MA, 1966).

[21] J. D. Huba, NRL Plasma Formulary, Naval Research Laboratory, NRL/PU/6790-04-477:36 (2004).
[22] V. Saxena and B. Ziaja, Hydrodynamic model for expansion and collisional relaxation of $\mathrm{x}$-ray laser-excited multi-component nanoplasma, Phys. Plasmas 23, 012710 (2016).

[23] W. L. Kruer, The Physics of Laser Plasma Interactions (Addison Wesley, Reading, MA, 1988).

[24] T. Ditmire, T. Donnelly, A. M. Rubenchik, R. W. Falcone, and M. D. Perry, Interaction of intense laser pulses with atomic clusters, Phys. Rev. A 53, 3379 (1996).

[25] I. Last and J. Jortner, Quasiresonance ionization of large multicharged clusters in a strong laser field, Phys. Rev. A 60, 2215 (1999).

[26] V. P. Krainov and M. B. Smirnov, Cluster beams in the super-intense femtosecond laser pulse, Phys. Rep. 370, 237 (2002).

[27] B. Ziaja, H. Wabnitz, F. Wang, E. Weckert, and T. Möller, Ionization and Expansion Dynamics of Atomic Clusters Irradiated with Short Intense VUV Pulses, Phys. Rev. Lett. 102, 205002 (2009).

[28] B. Ziaja et al., Emission of electrons from rare gas clusters after their irradiation with intense VUV pulses of $100 \mathrm{~nm}$ and $32 \mathrm{~nm}$ wavelength, New J. Phys. 11, 103012 (2009).

[29] R. R. Fäustlin et al., Observation of Ultrafast Nonequilibrium Collective Dynamics in a Warm Dense Hydrogen Plasma, Phys. Rev. Lett. 104, 125002 (2010).

[30] S.-K. Son and R. Santra, Monte Carlo calculation of ion, electron, and photon spectra of xenon atoms in x-ray freeelectron laser pulses, Phys. Rev. A 85, 063415 (2012).

[31] Z. Jurek, S.-K. Son, B. Ziaja, and R. Santra, XMDYN and XATOM: versatile simulation tools for quantitative modeling of X-ray free-electron laser induced dynamics of matter, J. Appl. Cryst. 49 (2016).

[32] S.-K. Son, L. Young, and R. Santra, Impact of hollow-atom formation on coherent $\mathrm{X}$-ray scattering at high intensity, Phys. Rev. A 83, 033402 (2011).

[33] S.-K. Son, R. Thiele, Z. Jurek, B. Ziaja, and R. Santra, Quantum mechanical calculation of ionization-potential lowering in dense plasmas, Phys. Rev. X 4, 031004 (2014).

[34] M. O. Krause and J. H. Oliver, Natural width of atomic K and L levels K $\alpha$ X-ray lines and several KLL Auger lines, J. Phys. Chem. Ref. Data 8, 329 (1979).

[35] A. C. Thompson et al., X-Ray Data Booklet, Center for X-ray Optics and Advanced Light Source, 2nd ed., Lawrence Berkeley Laboratory (University of California, 2001).

[36] M. A. Lennon et al., Recommended data on the electron impact ionization of atoms and ions: fluorine to nickel, J. Phys. Chem. Ref. Data 17, 1285 (1988).

[37] J. Oxenius, Kinetic Theory of Particles and Photons: Theoretical Foundations of Non-LTE Plasma Spectroscopy (Springer Verlag, Berlin, 1986), pp. 21-65. 